\title{
Differential effect of inbred mouse strain (C57BL/6, DBA/2, 129T2) on insulin secretory function in response to a high fat diet
}

\author{
Sofianos Andrikopoulos, Christine M Massa, \\ Kathryn Aston-Mourney, Alexandra Funkat, Barbara C Fam, \\ Rebecca L Hull ${ }^{1}$, Steven E Kahn ${ }^{1}$ and Joseph Proietto \\ University of Melbourne, Department of Medicine (AH/NH), Heidelberg Repatriation Hospital, Heidelberg Heights, Victoria 3081, Australia \\ ${ }^{1}$ Division of Metabolism, Endocrinology and Nutrition, Department of Medicine, VA Puget Sound Health Care System and University of Washington, Seattle, \\ Washington 98108, USA \\ (Requests for offprints should be addressed to S Andrikopoulos, University of Melbourne, Department of Medicine (AH/NH), Heidelberg Repatriation Hospital, \\ 300 Waterdale Road, Heidelberg Heights, Victoria 3081, Australia; Email: sof@unimelb.edu.au)
}

\begin{abstract}
The increasing production of genetically-modified mouse models has necessitated studies to determine the inherent physiological characteristics of commonly used mouse strains. In this study we examined insulin secretory function in response to an intravenous bolus of glucose or glucose plus arginine in anesthetized C57BL/6, DBA/2 and $129 \mathrm{~T} 2$ mice fed either a control or high fat diet for 6 weeks. The results show that $129 \mathrm{~T} 2$ mice had higher fasting plasma glucose levels and lower fasting plasma insulin levels compared with C57BL/6 and DBA/2 mice regardless of diet. Furthermore, 129T2 mice were glucose intolerant and secreted significantly less insulin in response to glucose and glucose plus arginine irrespective of diet compared with the other two strains of mice. DBA/2 mice hypersecreted insulin in response to glucose and glucose
\end{abstract}

plus arginine compared with C57BL/6 and 129T2 mice. Moreover while first phase insulin secretion was appropriately increased in response to the high fat diet in C57BL/6 and $129 \mathrm{~T} 2$ mice, this was not the case for DBA/2 mice. Mean islet area was decreased in response to a high fat diet in DBA/2 mice, while there was no dietary effect on the other two strains. This study highlights the inherent genetic differences that exist among seemingly normal strains of mice that are commonly used to make transgenic and knockout mice. Understanding these differences will provide researchers with the information to choose the appropriate genetic background on which to express their particular genetic alteration.

Journal of Endocrinology (2005) 187, 45-53

\section{Introduction}

Type 2 diabetes is characterized by fasting hyperglycemia, which is contributed to by reduced glucose-mediated insulin secretion and a reduction in the efficiency of glucose uptake by the insulin sensitive tissues. While mechanisms that are responsible for the impairments in insulin release and insulin sensitivity in type 2 diabetes are not fully known, it is generally accepted that multiple genetic defects are required in the presence of a permissive environment (Kahn 2003a). For example, obesity or energy-rich (high fat) diets can exacerbate metabolic defects associated with type 2 diabetes, such as an impairment in insulin secretion (Lee et al. 1995, Paolisso et al. 1995, Zraika et al. 2002, Zraika et al. 2004) and the development of insulin resistance (Shulman 2000, Hotamisligil 2000, Song et al. 2001, Lamont et al. 2003).

To assess the impact of a particular genetic defect on glucose metabolism and the development of type 2 dia- betes, it is has become increasingly popular to under- or over-express candidate genes by generating geneticallymodified mouse models (Valet et al. 2002, Kahn 2003b). However, there are now a number of reports that demonstrate that the same genetic alteration results in profoundly different phenotypes when present on different mouse strain backgrounds. Examples of these include embryonic lethality versus normal viable mice (Casademunt et al. 1999), premature death versus a normal longevity (Al-Saktawi et al. 2003) and inflammatory diseases versus no evidence of such (Murata et al. 2002). These examples are just a few that highlight the importance of the mouse strain on the resultant phenotype of a particular genetic manipulation.

When one considers obesity and diabetes, the effect of genetic background was recognized 30 years ago when it was shown that expression of either the leptin gene $(o b / o b)$ or the leptin receptor gene $(d b / d b)$ mutation on the C57BL/6 background resulted in a phenotype of massive 
obesity accompanied by insulin resistance with only transient diabetes, while the same mutations produced initial obesity and insulin resistance followed by life shortening diabetes when present on the C57BL/KsJ strain (Hummel et al. 1972, Coleman \& Hummel 1973, Leiter et al. 1981). Furthermore, reduction of $\beta$-cell glucokinase levels resulted in glucose intolerance in C57BL/6 but not $\mathrm{C} 3 \mathrm{H}$ mice (Ishihara et al. 1995), while the absence of the insulin receptor caused severe hyperinsulinemia on the Sv129 background strain compared with the C57BL/6 background strain (Kido et al. 2000). More recently, it has been shown that the double heterozygous knockout for the insulin receptor and insulin receptor substrate-1 resulted in marked hyperinsulinemia, islet hyperplasia and diabetes on the C57BL/6 background, while mild hyperinsulinemia, minimal islet hyperplasia and virtually no diabetes occurred on the Sv129 strain (Almind et al. 2003, Kulkarni et al. 2003). Finally, it has been suggested that the development of diabetes in a subpopulation of mice as a result of insulin receptor substrate-2 ablation is due to modifier genes from the Sv129, but not CBA, strains (Terauchi et al. 2002). The field is further complicated since the use of the Sv129 nomenclature referred to a number of substrains including 129S4, 129S6, 129T2, 129X1 and others (Simpson et al. 1997).

It is therefore evident that to understand the resultant phenotype of a specific genetic manipulation the inherent phenotype of the particular mouse strain needs to be known. Although it is now becoming recognized that different inbred strains harbor different susceptibilities for either obesity or diabetes, knowledge of insulin secretory function and $\beta$-cell secretory capacity, particularly in response to a high fat diet, of most if not all strains is lacking. The aim of this study, therefore, was to determine the effect of mouse strain on glucose-induced insulin release as well as $\beta$-cell secretory capacity. To address this, we studied three of the most commonly used inbred mouse strains namely, C57BL/6, DBA/2 and 129T2 and examined insulin release in response to glucose and glucose plus arginine in mice on low and high fat diets.

\section{Materials and Methods}

\section{Mice and diets}

Eight-week old C57BL/6, DBA/2 and 129T2 (formerly known as 129T2/SvEmsJ (Simpson et al. 1997)) mice were obtained from the Walter and Eliza Hall Institute of Medical Research Animal facility (Kew, Vic, Australia) and housed while consuming a diet of standard laboratory chow containing (w/w) 6\% fat, 20\% protein and 74\% carbohydrate (Barastoc, Pakenham, Vic, Australia) with water provided ad libitum. Only male mice were used in this study, thus avoiding any influence of ovarian hormones and cycling on insulin secretion that might have occured if female mice were used. It is also important to note that the phenotype of the males is more severe in many animal models of obesity and insulin resistance. Lighting was artificial and maintained to a 12-hr daynight cycle (lights on at $0600 \mathrm{~h}$ ). The room temperature was kept constant at $22^{\circ} \mathrm{C}$. At 10 weeks of age the mice were randomly divided into the two diet groups. The control group received a diet containing (w/w) 7\% fat, $18 \%$ protein and $75 \%$ carbohydrate. The high fat group received adiet containing (w/w) $60 \%$ fat, $18 \%$ protein and $22 \%$ carbohydrate. Both diets were otherwise identical and manufactured by Glen Forrest Stockfeeders, Glenn Forrest, WA, Australia. The composition of the diets has been previously reported (Funkat et al. 2004). Mice were housed in pairs and body weights were determined weekly for five weeks. In the sixth week mice were subjected to intravenous glucose or glucose plus arginine tolerance tests as described below. Ethics approval was obtained from Melbourne Health Animal Ethics Committee and all experiments were carried out in accordance with the guidelines stipulated by this board.

\section{Intravenous glucose and glucose plus arginine tolerance tests}

The intravenous glucose and glucose plus arginine tolerance tests were performed $(n=10$ per test group for each test) following six weeks of diet as previously described (Pacini \& Ahren 1999, Kooptiwut et al. 2002) with the following modifications. Glucose plus arginine administration was used to determine $\beta$-cell capacity for insulin secretion. Animals were studied at $0900 \mathrm{~h}$ after an overnight fast of $17 \mathrm{~h}$ (food withdrawn at $1600 \mathrm{~h}$ the previous day) and anesthetized with an intraperitoneal injection $(100 \mathrm{mg} / \mathrm{kg})$ of sodium pentobarbitone (Nembutal, Rhone Merieux, QLD, Australia). A silastic catheter (0.012" internal diameter, 0.025" outer diameter, Dow Corning, Midland MI, USA) filled with heparinized saline $(20 \mathrm{U} / \mathrm{ml})$ was inserted into the left carotid artery and the animals were allowed to recover from the surgery for $20 \mathrm{~min}$. Animals were kept warm with a heat lamp and body temperature was monitored using a rectal probe. A bolus of glucose $(1 \mathrm{~g} / \mathrm{kg})$ or glucose plus arginine $(1 \mathrm{~g} / \mathrm{kg}$ of each) was injected through the carotid artery and $200 \mu \mathrm{l}$ of blood was sampled at $0,1,5,10,20,30$ and $50 \mathrm{~min}$ for plasma glucose and insulin analyses. Blood was immediately centrifuged, the plasma separated and the red blood cells resuspended in an equal volume of heparinized saline and reinfused into the animal via the carotid artery prior to the collection of the next blood sample to prevent anaemic shock.

\section{Tissue collection}

At the end of the tolerance test mice were euthanized with an overdose of sodium pentobarbitone and epididymal adipose tissues were removed and wet mass was determined. As the same person performed the procedure on all 
Table 1 Fasting body weights $(\mathrm{g})$, plasma glucose $(\mathrm{mmol} / \mathrm{l})$ and plasma insulin $(\mathrm{ng} / \mathrm{ml})$ concentrations of all mice fed a control or high fat diet for six weeks

\section{Control Diet}

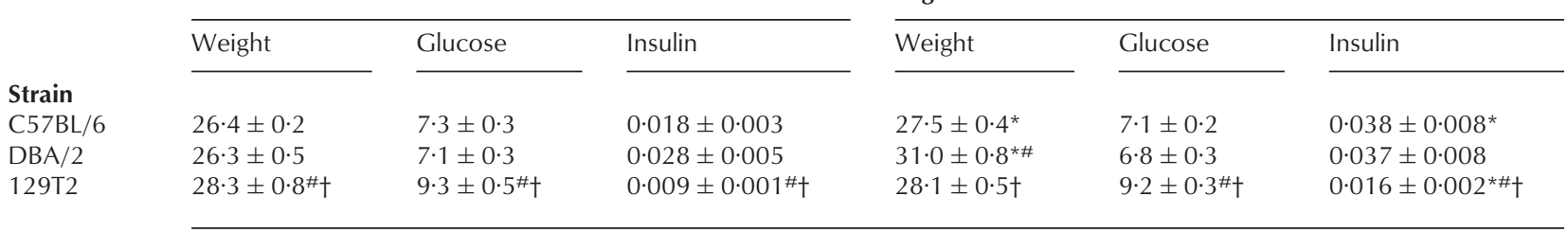

Values are presented as means \pm S.E.M. $(n=20) .{ }^{*} P<0.05$ vs control diet; ${ }^{\#} P<0.05$ vs $C 57 \mathrm{BL} / 6 ;+P<0.05$ vs DBA/2. mice the dissection of the adipose tissue depots was standardized, thus reducing the potential for variability.

\section{Measurement of plasma glucose and insulin levels}

The glucose oxidase method was used to determine plasma glucose using a GM7 Analox glucose analyzer (Helena Laboratories, Mount Waverley Vic, Australia). Plasma insulin was determined by a commercially available sensitive radioimmunoassay specific for rodent insulin (Linco Research Immunoassay, St Charles, MO, USA).

\section{Pancreatic histology}

A portion of the pancreas from each mouse was fixed overnight in phosphate-buffered paraformaldehyde $(4 \%$ $\mathrm{w} / \mathrm{v})$, dehydrated and embedded in paraffin. Five $\mathrm{mm}$ thick sections were stained with anti-insulin antibody to visualize islets and counterstained with the nuclear stain Hoechst 33342 and thioflavin S to aid morphological visualization of islets. Antibodies, Hoechst 33342 and thioflavin $\mathrm{S}$ were obtained from Sigma. Islet area was determined by manually circumscribing the outline of an average of 13 islets per mouse on the thioflavin S channel, where the outline of the islets was clearly visible and computing the outlined area using the Image Pro Plus (Media Cybernetics, Inc., Silver Spring, MD, USA).

\section{Statistical analysis}

Data are expressed as means \pm S.E.M. Comparisons among the three strains and the two diets were made by using a 2-way ANOVA with the Tukey's HSD post hoc test (SPSS 11 for Windows; SPSS Inc., Chicago, IL, USA). The level of statistical significance was set at $P<0 \cdot 05$.

\section{Results}

Body weight gain and fat pad weight following a control and high fat diets

Fasting body weight was approximately 7\% higher in 129 T2 mice compared with the other two strains following six weeks on the control diet, while DBA/2 mice were
$10 \%$ heavier following the high fat diet compared with the other two strains (Table 1). The gain in body weight over five weeks was significantly higher in DBA/2 mice compared with C57BL/6 and 129T2 mice fed the control diet (Figure 1A). Likewise, a high fat diet resulted in significantly increased body weight gain in $\mathrm{DBA} / 2$ mice compared with C57BL/6 and 129T2 mice fed the same high fat diet (Figure 1B). High fat feeding also resulted in increased body weight and weight gain in C57BL/6 and $\mathrm{DBA} / 2$ but not $129 \mathrm{~T} 2$ mice when these groups of mice were compared with the same genetic background that were consuming the control diet (Table 1 and Figure 1B).

The epididymal fat pad was heavier in DBA/2 mice compared with the other two strains regardless of the diet (Figure 1C). Again, while a high fat diet resulted in heavier epididymal fat pads in $\mathrm{C} 57 \mathrm{BL} / 6$ and $\mathrm{DBA} / 2$ mice, no change in epididymal fat pad mass was seen in 129T2 mice when compared with the control fed counterparts (Figure 1C). The weights of other fat pads (perinephric, subcutaneous) reflected the changes seen in the epididymal fat pads (data not shown).

Plasma glucose and insulin levels during the intravenous glucose tolerance tests (IVGTT)

Fasting plasma glucose levels were approximately $2 \mathrm{mmol} / 1$ higher in 129T2 mice compared with the other two strains on either the control or high fat diets (Table 1). However, high fat feeding for six weeks was not associated with changes in fasting plasma glucose levels within strains (Table 1).

Following the intravenous injection of the glucose bolus, control fed 129T2 mice displayed significantly higher plasma glucose levels compared with control fed C57BL/6 and DBA/2 mice (Figure 2A). Similarly, following the glucose bolus high fat fed 129T2 mice demonstrated significantly higher plasma glucose levels compared with high fat fed C57BL/6 and DBA/2 mice (Figure 2B). Consequently, the area under the glucose curve was $15 \%$ and $30 \%$ greater in $129 \mathrm{~T} 2$ mice compared with the other two strains, following the control and high fat diets respectively (Figure 2C).

Fasting plasma insulin levels were 50\%-70\% lower in $129 \mathrm{~T} 2$ mice compared with C57BL/6 and DBA/2 mice 
$\mathbf{A}$



B



$\mathbf{C}$

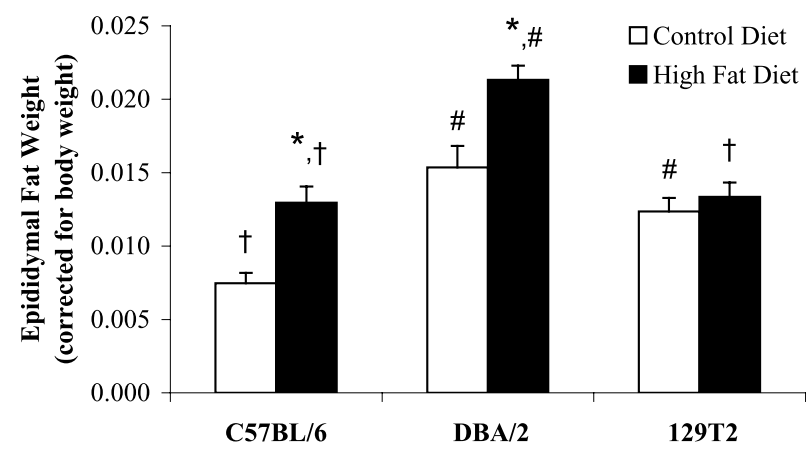

Figure 1 Body weight gain in mice fed a control diet (A) or a high fat diet (B) for five weeks. (C) Epididymal fat pad weights from mice fed a control or high fat diet. Values are presented as means \pm S.E.M. $(n=10) .{ }^{*} P<0.05$ vs control diet; $\# P<0.05$ vs $C 57 \mathrm{BL} / 6$; $+P<0 \cdot 05$ vs $\mathrm{DBA} / 2$.

irrespective of diet (Table 1). A high fat diet resulted in approximately 2-fold higher fasting plasma insulin levels in C57BL/6 and 129T2 mice compared with their control fed counterparts (Table 1).

Following the intravenous injection of glucose, plasma insulin levels were profoundly and significantly lower in $129 \mathrm{~T} 2$ mice compared with C57BL/6 and DBA/2 mice at most time-points irrespective of diet (Figure $3 \mathrm{~A}$ and $\mathrm{B}$ ). Control fed DBA/2 mice displayed higher plasma insulin levels at 1,5 and $10 \mathrm{~min}$ compared with control fed C57BL/6 mice (Figure 3A). Interestingly only C57BL/6
A

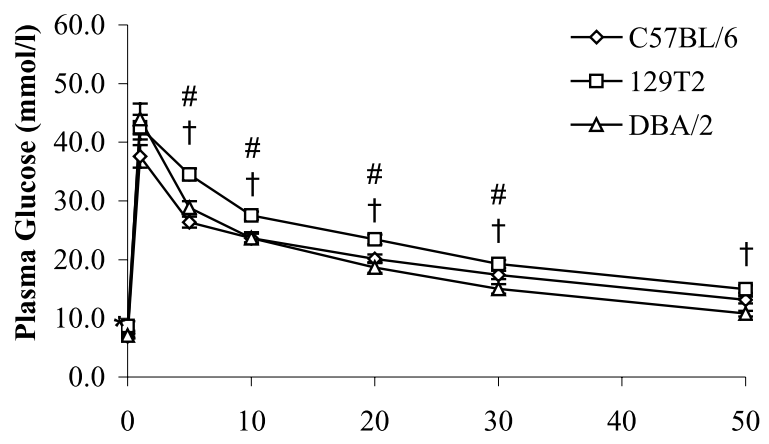

Time (mins)
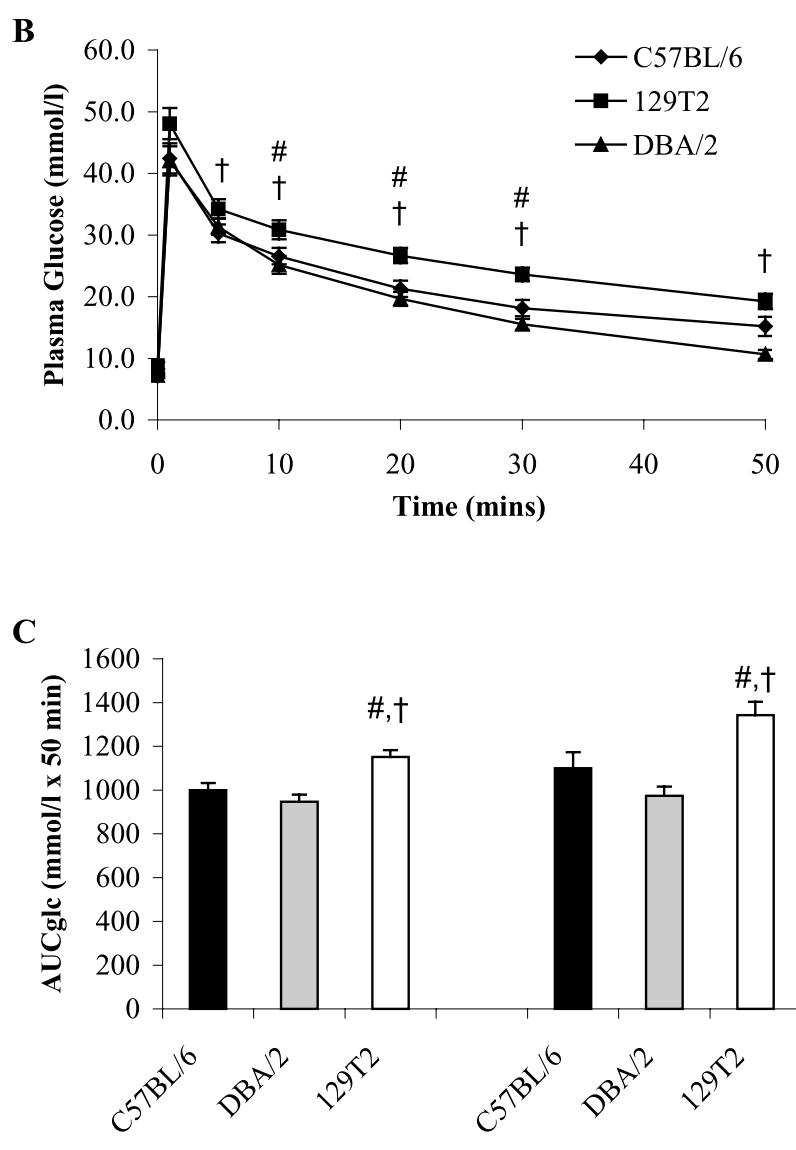

\section{Control Diet}

High Fat Diet

Figure 2 Plasma glucose concentrations during the intravenous glucose tolerance test $(1 \mathrm{~g} / \mathrm{kg})$ in mice fed a control diet (A) or a high fat diet (B) for six weeks. (C) Total area under the glucose curve (AUCglc) in mice fed a control or high fat diet. Values are presented as means \pm S.E.M. $(n=10)$. ${ }^{*} P<0.05$ vs control diet; $\# P<0 \cdot 05$ vs $\mathrm{C} 57 \mathrm{BL} / 6 ;+P<0 \cdot 05$ vs $\mathrm{DBA} / 2$.

mice showed a $40 \%$ increase in plasma insulin levels during the IVGTT when fed a high fat diet compared with their control fed counterparts (Figure 3B). 

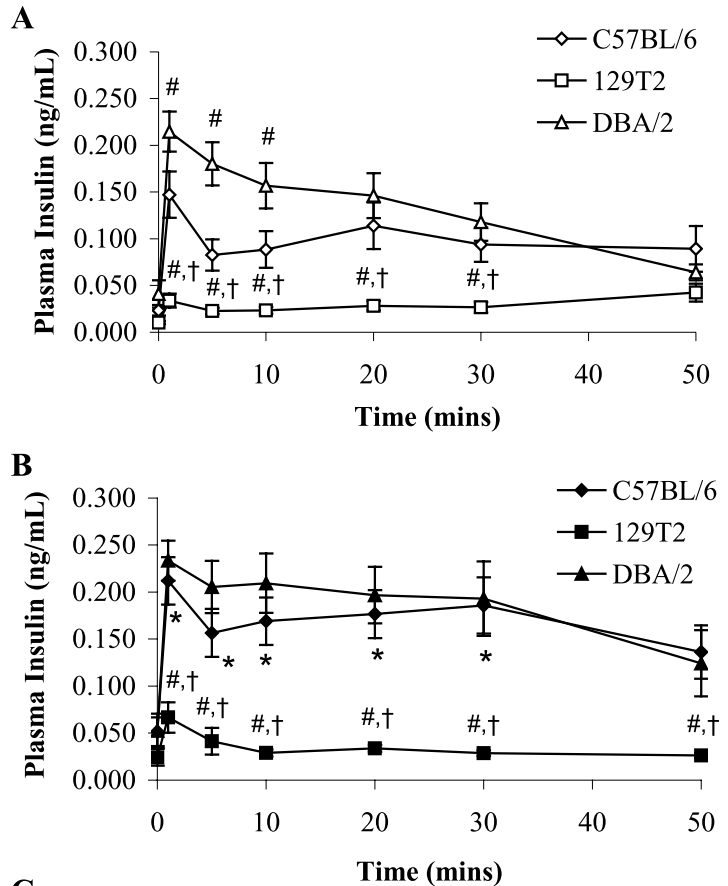

C

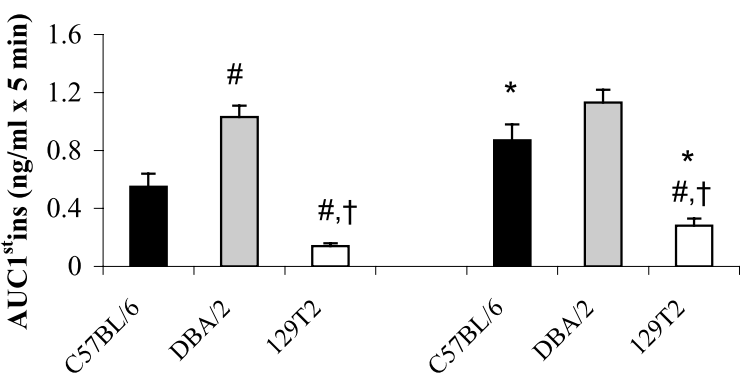

D Control Diet

High Fat Diet



Control Diet

High Fat Diet

Figure 3 Plasma insulin concentrations during the intravenous glucose tolerance test $(1 \mathrm{~g} / \mathrm{kg})$ in mice fed a control diet (A) or a high fat diet (B) for six weeks. (C) Area under the insulin curve for the first five min (AUC1 ${ }^{\text {st }}$ ins) representing first phase insulin secretion in mice fed a control or high fat diet. (D) Total area under the insulin curve (AUCins) in mice fed a control or high fat diet. Values are presented as means \pm S.E.M. $(n=10)$. ${ }^{*} P<0.05$ vs control diet; $\# P<0.05$ vs $C 57 \mathrm{BL} / 6 ;+P<0.05$ vs $\mathrm{DBA} / 2$.
First-phase insulin secretion (AUC1 $1^{\text {stins) }}$ was assessed by the area under the curve between 0 and 5 min post glucose challenge and was 70\%-85\% lower in $129 \mathrm{~T} 2$ mice compared with $\mathrm{C} 57 \mathrm{BL} / 6$ and $\mathrm{DBA} / 2$ mice irrespective of diet (Figure 3C). AUC1 ${ }^{\text {st }}$ ins was 2-fold higher in DBA/2 compared with C57BL/ 6 mice fed a control diet. A high fat diet resulted in an increase in AUC1 $1^{\text {st }}$ ins in $\mathrm{C} 57 \mathrm{BL} / 6$ and 129T2 but not DBA/2 mice (Figure 3C).

In agreement, total insulin secretion (AUCins) following the glucose challenge was also 70-85\% lower in $129 \mathrm{~T} 2$ mice compared with the other two strains regardless of diet (Figure 3D). DBA/2 mice fed the control diet displayed a $30 \%$ higher AUCins compared with C57BL/6 mice. A high fat diet resulted in a $40 \%$ and $30 \%$ increase in AUCins in C57BL/6 and DBA/2 but not $129 \mathrm{~T} 2$ mice (Figure 3D).

Plasma glucose and insulin levels during the intravenous glucose plus arginine tolerance tests

Plasma glucose levels were lower in DBA/2 mice compared with C57BL/6 and $129 \mathrm{~T} 2$ mice fed a control diet (Figure 4A). In response to a high fat diet DBA/2 mice had lower plasma glucose levels at 20, 30 and $50 \mathrm{~min}$ compared with C57BL/6 mice and at 30 and $50 \mathrm{~min}$ compared with $129 \mathrm{~T} 2$ mice (Figure 4B). Consequently, area under the glucose curve (AUCglc) was 30-40\% lower in the DBA/2 mice compared with the other two strains irrespective of diet (Figure 4C).

In response to an intravenous bolus of glucose plus arginine plasma insulin levels were higher in DBA/2 mice compared with the other two strains, irrespective of diet (Figure 5A and B). A high fat diet caused increased plasma insulin levels at 20,30 and $50 \mathrm{~min}$ in DBA/2 mice and at most time points in $129 \mathrm{~T} 2$ mice compared with their respective control fed counterparts (Figure 5B).

Consequently, AUC1 ${ }^{\text {st }}$ ins and AUCins in response to a glucose plus arginine bolus were approximately $20 \%$ and $40 \%$ higher in the DBA/2 mouse compared with the other two strains, irrespective of the diet (Figure 5C and D). Interestingly, compared with their control fed counterparts a high fat diet caused a $20 \%$ and $30 \%$ increase in AUC $1^{\text {st }}$ ins and AUCins respectively only in the $129 \mathrm{~T} 2$ strain (Figure 5C and D). A high fat diet so resulted in $35 \%$ increase in AUCins in DBA/2 mice compared with their control fed counterparts (Figure 5D).

\section{Pancreatic insulin content}

Pancreatic insulin content was not different among the three strains of mice fed the control diet at the conclusion of either the intravenous glucose or glucose plus arginine tolerance tests (Figure 6A and B). A high fat diet was associated with a $60 \%$ and $40 \%$ reduction in insulin content in C57BL/6 and 129T2 mice following the glucose bolus (Figure 6A). Insulin content was also 70\% 
A

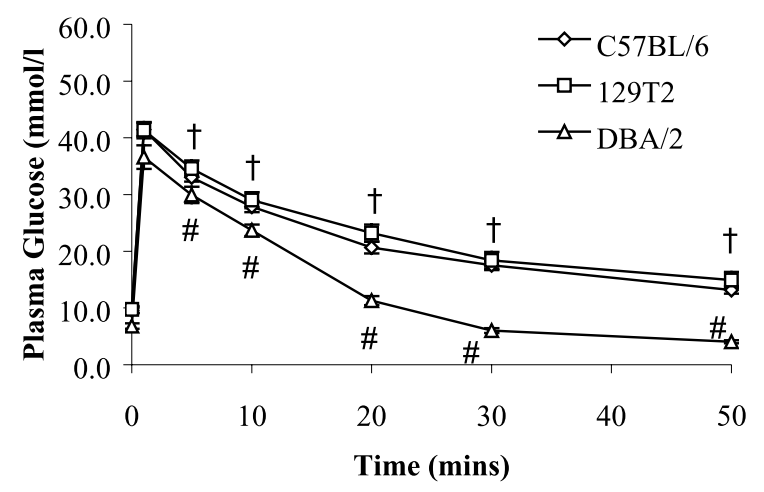

B

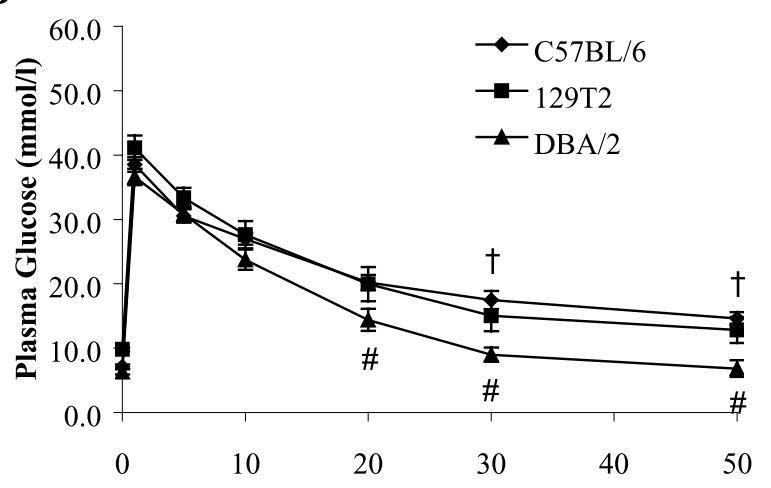

C

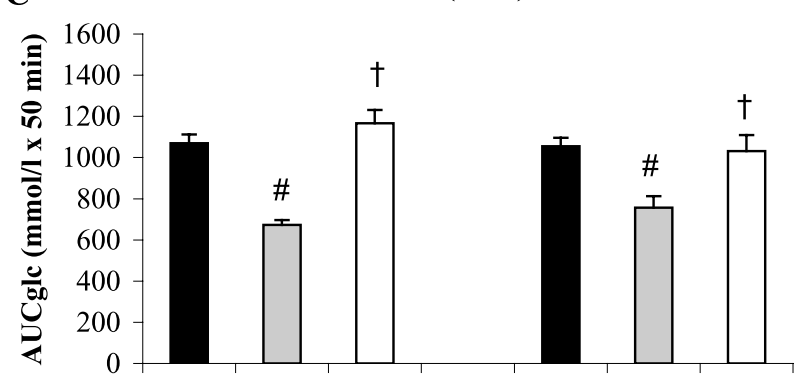

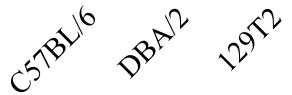

Control Diet

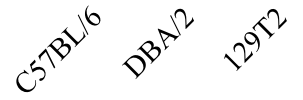

High Fat Diet
Figure 4 Plasma glucose concentrations during the intravenous glucose plus arginine tolerance test $(1 \mathrm{~g} / \mathrm{kg}$ of each) in mice fed a control diet (A) or a high fat diet (B) for six weeks. (C) Total area under the glucose curve (AUCglc) in mice fed a control or high fat diet. Values are presented as means \pm S.E.M. $(n=10) .{ }^{*} P<0.05$ vs control diet; $\# P<0.05$ vs $C 57 \mathrm{BL} / 6 ;+P<0 \cdot 05$ vs DBA $/ 2$.

reduced in $\mathrm{C} 57 \mathrm{BL} / 6$ mice following the glucose plus arginine bolus (Figure 6B). At the end of the glucose plus arginine test, insulin content was 2-fold higher in high fat fed compared with control fed DBA/2 mice (Figure 6B). Insulin content was lower irrespective of strain and diet
A

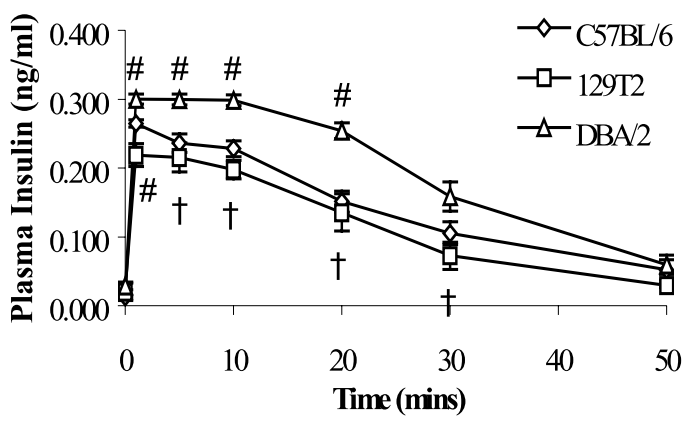

B

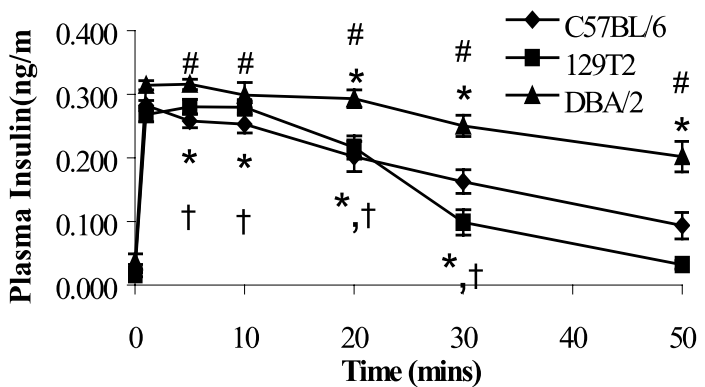

C



D

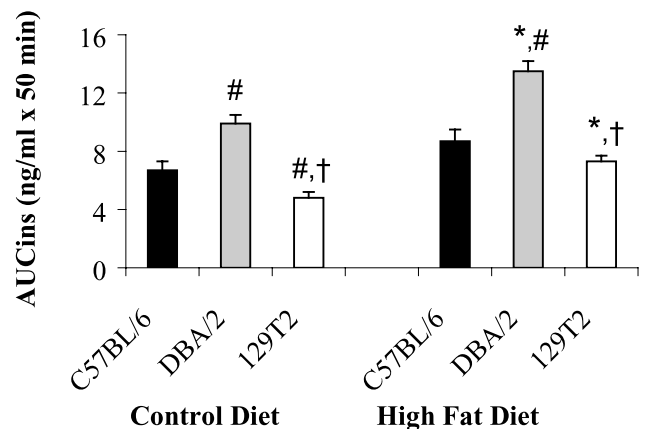

Figure 5 Plasma insulin concentrations during the intravenous glucose plus arginine tolerance test $(1 \mathrm{~g} / \mathrm{kg}$ of each) in mice fed a control diet (A) or a high fat diet (B) for six weeks. (C) Area under the insulin curve for the first five min ( $A \cup C 1^{\text {st }}$ ins) representing first phase insulin secretion in mice fed a control or high fat diet. (D) Total area under the insulin curve (AUCins) in mice fed a control or high fat diet. Values are presented as means \pm S.E.M. $(n=10)$. ${ }^{*} P<0.05$ vs control diet; $\# P<0.05$ vs $C 57 \mathrm{BL} / 6 ;+P<0.05$ vs $\mathrm{DBA} / 2$. 

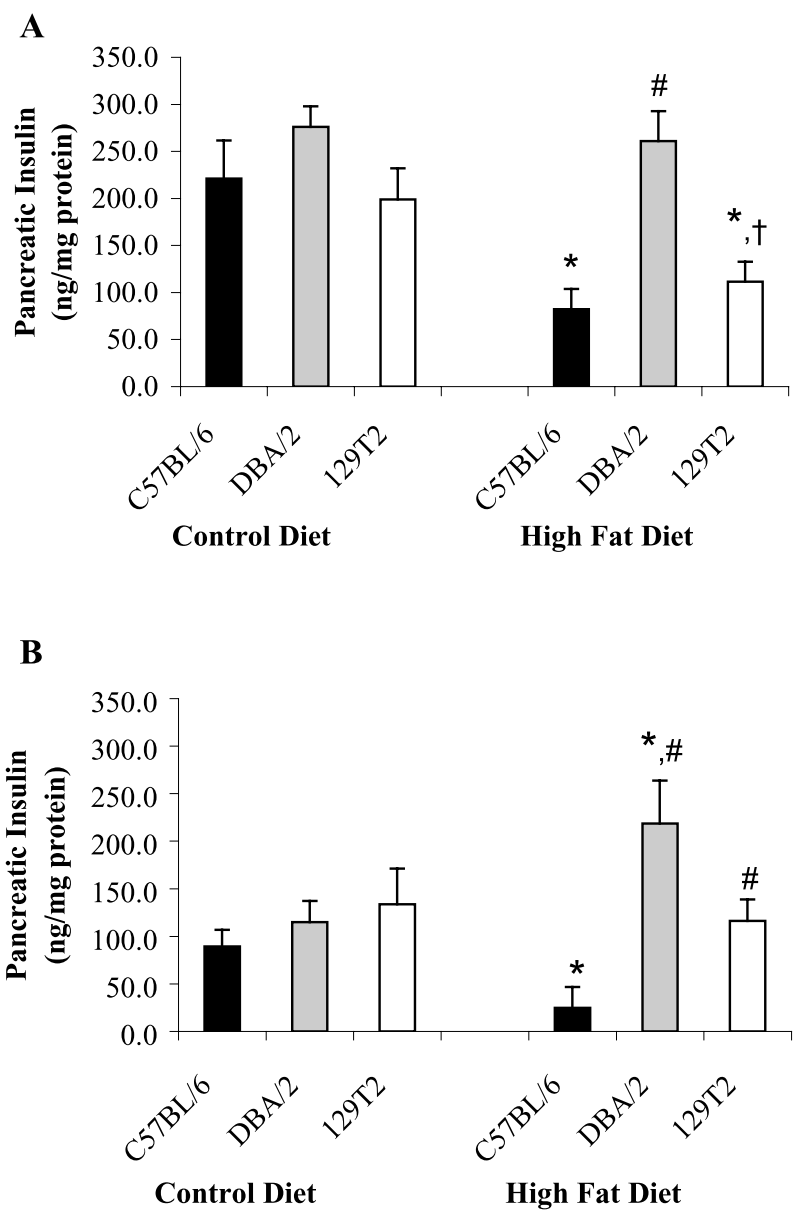

Figure 6 Pancreatic insulin content in mice fed a control diet or a high fat diet following (A) the intravenous glucose or (B) the intravenous glucose plus arginine tolerance test. Values are presented as means \pm S.E.M. $(n=10)$. ${ }^{*} P<0 \cdot 05$ vs control diet; $\# P<0.05$ vs $\mathrm{C} 57 \mathrm{BL} / 6 ;+P<0.05$ vs $\mathrm{DBA} / 2$.

following the glucose plus arginine compared with glucose alone.

\section{Pancreatic islet area}

Mean islet area was significantly higher in DBA/2 mice compared with the other two strains following a control $\operatorname{diet}\left(12881 \pm 1150 \mu \mathrm{m}^{2}\right.$ vs $5550 \pm 778 \mu \mathrm{m}^{2}$ and 7906 $\pm 1335 \mu^{2}$, DBA $/ 2$ vs C57BL/ 6 and $129 \mathrm{~T} 2, n=6$, $P<0 \cdot 05)$. Interestingly, a high fat diet was associated with reduced islet area in DBA/2 mice only compared with their control fed counterparts $\left(6905 \pm 1227 \mu \mathrm{m}^{2}\right.$ vs $\left.12881 \pm 1150 \mu \mathrm{m}^{2}, n=6, \quad P<0 \cdot 05\right)$. As a result there was no difference in islet area among the three strains of mice fed the high fat diet $\left(6665 \pm 1061 \mu \mathrm{m}^{2}, 6905\right.$ $\pm 1227 \mu \mathrm{m}^{2}, 8277 \pm 1308 \mu \mathrm{m}^{2}, \mathrm{C} 57 \mathrm{BL} / 6, \mathrm{DBA} / 2$ and 129T2 respectively).

\section{Discussion}

A powerful and frequently utilized approach to determe the importance of a specific gene in the pathology of disease (for example, obesity and diabetes) is to produce genetically altered animal models that over- or underexpress the gene(s) of interest, either globally or in a specific tissue. However, it has recently become increasingly recognized that genetic background can result in a different phenotype when the same genetic manipulation is applied. Furthermore, for a number of reasons, including the need to increase hybrid vigor as well as the increasing use of the Cre/LoxP system (which requires a tissuespecific Cre expressing and a LoxP-containing gene targeted mouse), the resultant genetically modified mouse is frequently a conglomeration of two or more strains. This warrants studies on the inherent physiological and biochemical parameters of commonly used mouse strains to provide a more informed choice on the background strain on which a genetic manipulation should be placed. While there have recently been a number of studies investigating the inherent metabolic characteristics of commonly used inbred strains of mice (Kooptiwut et al. 2002, Almind et al. 2003, Kulkarni et al. 2003, Rossmeisl et al. 2003, Almind \& Kahn 2004, Goren et al. 2004, Bock et al. 2005), the present study is the first to assess insulin secretion in vivo using the intravenous administration of stimuli and to determine islet area in response to a high fat diet. Furthermore, this is the first study to use the $129 \mathrm{~T} 2$ substrain, which has been used in the generation of gene knockout mice (Chong et al. 2002, Kaczmarczyk et al. 2003).

An important finding of our study was that the $129 \mathrm{~T} 2$ strain secreted significantly less insulin when challenged with a glucose bolus compared with the C57BL/6 and $\mathrm{DBA} / 2$ strains, regardless of the fat content of the diet. This was reflected in both a decreased first phase response and a reduced response to glucose plus arginine, the latter providing an estimate of $\beta$-cell secretory capacity. Furthermore, 129T2 mice displayed higher plasma glucose levels during the IVGTT, indicative of diminished glucose tolerance. That this change in glucose tolerance is the result of the diminished insulin response, likely being due to a reduction in $\beta$-cell function rather than simply a difference in insulin sensitivity or a change in islet size, is suggested by the observations of the fasting glucose and insulin levels. The lower fasting insulin levels are in keeping with the $129 \mathrm{~T} 2$ mice being more insulin sensitive. However, the elevated fasting glucose levels, along with the greater glucose excursion following glucose administration, suggest otherwise and are in keeping with the concept of reduced $\beta$-cell responsiveness as the basis for the reduction in the efficiency of glucose disposal. The above data are in contrast to a recent study showing that $129 \mathrm{X} 1$ mice had lower fasting plasma glucose levels and better glucose tolerance than C57BL/6 mice (Goren et al. 
2004). The reason for the differences between our and this study is likely due to the different substrains (129T2 vs 129X1) that were examined. Interestingly, in response to glucose plus arginine, $129 \mathrm{~T} 2$ mice displayed a significant increase in insulin secretion similar to that seen in C57BL/6 mice but still lower than DBA/2 mice. A similar pattern was also displayed by 129X1 mice (Goren et al. 2004), which showed a grossly blunted insulin secretory response to glucose but released significantly more insulin in response to arginine. These results may suggest that the 129 substrains may not respond to glucose as an insulin secretagogue to the same extent as other mouse strains (C57BL/6, DBA/2), but do respond to other stimuli such as arginine.

As we have previously shown (Kooptiwut et al. 2002), first phase and total insulin secretion was higher in DBA/2 compared with C57BL/6 mice in response to glucose and glucose plus arginine. This is contrary to a study showing that there was no difference between $\mathrm{DBA} / 2$ and C57BL/6 mice in early phase insulin release (Goren et al. 2004). While we cannot be certain of the exact reason for these differences, it is possibly due to the different in vivo methods that were used to assess insulin secretion. In this study we administered glucose intravenously at $1 \mathrm{~g} / \mathrm{kg}$ body weight to anesthetized animals, while the Goren study (Goren et al. 2004) administered glucose intraperitoneally at $3 \mathrm{~g} / \mathrm{kg}$ body weight to conscious mice. In the current study the mice were anesthetized and had undergone surgery to place a carotid artery catheter before experimentation. Whether the intraperitoneal route of administration and state of consciousness is associated with the effect of factors that influence insulin release independent of glucose cannot be dismissed. However, our current observations of increased insulin release are in keeping with our previous reported finding that isolated islets from DBA/2 mice hypersecrete insulin in comparison to C57BL/6 mouse islets (Kooptiwut et al. 2002).

We also evaluated the effect of dietary fat and genetic background on the insulin response to classical $\beta$-cell secretagogues. In this assessment we found that on a high fat diet both C57BL/6 and 129T2 mice showed an increase in the first phase insulin response to glucose, while only the $129 \mathrm{~T} 2$ mice displayed an increase in the insulin response to glucose plus arginine. By contrast, high fat feeding did not result in an increase in first phase secretion in DBA/2 mice. Interestingly, mean islet area was reduced only in DBA/2 mice following the high fat diet. It is important to note that the dietary intervention in this study was of a relatively short term (6 weeks) and did not result in profound insulin secretory dysfunction as has previously been shown when mice were fed a high fat diet for 12 weeks or longer (Lee et al. 1995, Ahren et al. 1997, Surwit et al. 1988). It was therefore of interest to note that first phase secretion was not able to increase and it was interesting to observe the decrease in islet area in response to the high fat diet in DBA/2 mice. This data supports the idea that the DBA/2 strain may be more prone to islet dysfunction as has previously been shown by the expression of the leptin receptor mutation $(d b / d b)$ (Leiter et al. 1981) or when isolated islets were chronically cultured in a high glucose milieu (Pehuet-Figoni et al. 1994).

It is noteworthy that DBA/2 mice gained the most amount of weight and had the heaviest fat pads compared with the other two strains of mice regardless of diet. Furthermore, while C57BL/6 and DBA/2 mice gained weight following a high fat diet this was not the case for the 129T2 strain. Our laboratory has recently shown that DBA/2 mice display decreased physical activity and resting energy expenditure compared with C57BL/6 mice, which would partly explain the increase in fat mass (Funkat et al. 2004).

In conclusion, we show that there are marked differences in the insulin secretion profile in three inbred strains of mice that are commonly used for the generation of genetically altered mouse models of diabetes and obesity. These findings therefore have a bearing on a number of aspects when considering the use of mouse models. Knowledge of these effects is important for interpretation of the phenotypes of genetically modified mouse models. They also provide investigators with information that could be useful when selecting the appropriate genetic background on which to undertake a particular genetic modification when attempting to study diabetes and obesity.

\section{Acknowledgements}

This study was supported by the National Health and Medical Research Council of Australia (project numbers 145769209001 and 209002 to J P and S A) and by the United States Department of Veterans Affairs, the American Diabetes Association and NIH grant DK17047. S A was supported by an R D Wright Biomedical Career Development Award from the National Health and Medical Research Council of Australia. We would like to thank Ms Joanne McKie and Ms Valentina Jovanovska for excellent technical assistance. The authors declare that there is no conflict of interest that would prejudice the impartiality of this scientific work.

\section{References}

Ahren B, Simonsson E, Scheurink AJ, Mulder H, Myrsen U \& Sundler F 1997 Dissociated insulinotropic sensitivity to glucose and carbachol in high-fat diet-induced insulin resistance in C57BL/6J mice. Metabolism 46 97-106.

Almind K \& Kahn CR 2004 Genetic determinants of energy expenditure and insulin resistance in diet-induced obesity in mice. Diabetes 53 3274-3285.

Almind K, Kulkarni RN, Lannon SM \& Kahn CR 2003 Identification of interactive loci linked to insulin and leptin in mice with genetic insulin resistance. Diabetes 52 1535-1543. 
Al-Saktawi K, McLaughlin M, Klugmann M, Schneider A, Barrie JA, McCulloch MC, Montague P, Kirkham D, Nave KA \& Griffiths IR 2003 Genetic background determines phenotypic severity of the Plp rumpshaker mutation. Journal of Neuroscience Research 72 12-24.

Bock T, Pakkenberg B \& Buschard K 2005 Genetic background determines the size and structure of the endocrine pancreas. Diabetes 54 133-137.

Casademunt E, Carter BD, Benzel I, Frade JM, Dechant G \& Barde YA 1999 The zinc finger protein NRIF interacts with the neurotrophin receptor p75(NTR) and participates in programmed cell death. Embo Journal 18 6050-6061.

Chong MM, Thomas HE \& Kay TW 2002 Suppressor of cytokine signaling-1 regulates the sensitivity of pancreatic beta cells to tumor necrosis factor. Journal of Biological Chemistry 277 27945-27952.

Coleman DL \& Hummel KP 1973 The influence of genetic background on the expression of the obese $(\mathrm{Ob})$ gene in the mouse. Diabetologia 9 287-293.

Funkat A, Massa CM, Jovanovska V, Proietto J \& Andrikopoulos S 2004 Metabolic adaptations of three inbred strains of mice (C57BL/6, DBA/2, and 129T2) in response to a high-fat diet. Journal of Nutrition 134 3264-3269.

Goren HJ, Kulkarni RN \& Kahn CR 2004 Glucose homeostasis and tissue transcript content of insulin signaling intermediates in four inbred strains of mice: C57BL/6, C57 BLKS/6, DBA/2, and 129X1. Endocrinology 145 3307-3323.

Hotamisligil GS 2000 Molecular mechanisms of insulin resistance and the role of the adipocyte. International Journal of Obesity and Related Metabolic Disorders 24 Suppl 4 S23-S27.

Hummel KP, Coleman DL \& Lane PW 1972 The influence of genetic background on expression of mutations at the diabetes locus in the mouse. I. C57BL-KsJ and C57BL-6J strains. Biochemical Genetics 7 1-13.

Ishihara H, Tashiro F, Ikuta K, Asano T, Katagiri H, Inukai K, Kikuchi M, Yazaki Y, Oka Y \& Miyazaki J 1995 Inhibition of pancreatic beta-cell glucokinase by antisense RNA expression in transgenic mice: mouse strain-dependent alteration of glucose tolerance. FEBS Letters 371 329-332.

Kaczmarczyk SJ, Andrikopoulos S, Favaloro J, Domenighetti AA, Dunn A, Ernst M, Grail D, Fodero-Tavoletti M, Huggins CE, Delbridge LM et al. 2003 Threshold effects of glucose transporter-4 (GLUT4) deficiency on cardiac glucose uptake and development of hypertrophy. Journal of Molecular Endocrinology 31 449-459.

Kahn SE 2003a The relative contributions of insulin resistance and beta-cell dysfunction to the pathophysiology of type 2 diabetes. Diabetologia 46 3-19.

Kahn CR $2003 b$ The Gordon Wilson Lecture. Lessons about the control of glucose homeostasis and the pathogenesis of diabetes from knockout mice. Transactions of the American Clinical and Climatological Association 114 125-148.

Kido Y, Philippe N, Schaffer AA \& Accili D 2000 Genetic modifiers of the insulin resistance phenotype in mice. Diabetes 49 589-596.

Kooptiwut S, Zraika S, Thorburn AW, Dunlop ME, Darwiche R, Kay TW, Proietto J \& Andrikopoulos S 2002 Comparison of insulin secretory function in two mouse models with different susceptibility to beta-cell failure. Endocrinology 143 2085-2092.

Kulkarni RN, Almind K, Goren HJ, Winnay JN, Ueki K, Okada T \& Kahn CR 2003 Impact of genetic background on development of hyperinsulinemia and diabetes in insulin receptor/insulin receptor substrate-1 double heterozygous mice. Diabetes 52 1528-1534.
Lamont BJ, Andrikopoulos S, Funkat A, Favaloro J, Ye JM, Kraegen EW, Howlett KF, Zajac JD \& Proietto J 2003 Peripheral insulin resistance develops in transgenic rats overexpressing phosphoenolpyruvate carboxykinase in the kidney. Diabetologia 46 1338-1347.

Lee SK, Opara EC, Surwit RS, Feinglos MN \& Akwari OE 1995 Defective glucose-stimulated insulin release from perifused islets of C57BL/6J mice. Pancreas 11 206-211.

Leiter EH, Coleman DL \& Hummel KP 1981 The influence of genetic background on the expression of mutations at the diabetes locus in the mouse. III. Effect of H-2 haplotype and sex. Diabetes 30 1029-1034.

Murata K, Nose M, Ndhlovu LC, Sato T, Sugamura K \& Ishii N 2002 Constitutive OX40/OX40 ligand interaction induces autoimmune-like diseases. Journal of Immunology 169 4628-4636.

Pacini G \& Ahren B 1999 Dose-related effects of GLP-1 on insulin secretion, insulin sensitivity, and glucose effectiveness in mice. American Journal of Physiology - Endocrinology and Metabolism 277 E996-E1004.

Paolisso G, Gambardella A, Amato L, Tortoriello R, D'Amore A, Varricchio M \& D'Onofrio F 1995 Opposite effects of short- and long-term fatty acid infusion on insulin secretion in healthy subjects. Diabetologia 38 1295-1299.

Pehuet-Figoni M, Ballot E, Bach JF \& Chatenoud L 1994 Aberrant function and long-term survival of mouse $\beta$-cells exposed in vitro to high glucose concentrations. Cell Transplantation 3 445-451.

Rossmeisl M, Rim JS, Koza RA \& Kozak LP 2003 Variation in type 2 diabetes-related traits in mouse strains susceptible to diet-induced obesity. Diabetes 52 1958-1966.

Shulman GI 2000 Cellular mechanisms of insulin resistance. Journal of Clinical Investigation 106 171-176.

Simpson EM, Linder CC, Sargent EE, Davisson MT, Mobraaten LE \& Sharp JJ 1997 Genetic variation among 129 substrains and its importance for targeted mutagenesis in mice. Nature Genetics 16 $19-27$.

Song S, Andrikopoulos S, Filippis C, Thorburn AW, Khan D \& Proietto J 2001 Mechanism of fat-induced hepatic gluconeogenesis: effect of metformin. American Journal of Physiology - Endocrinology and Metabolism 281 E275-E282.

Surwit RS, Kuhn CM, Cochrane C, McCubbin JA \& Feinglos MN 1988 Diet-induced type II diabetes in C57BL/6J mice. Diabetes 37 $1163-1167$.

Terauchi Y, Matsui J, Suzuki R, Kubota N, Komeda K, Aizawa S, Eto K, Kimura S, Tobe K, Lienhard GE et al. 2002 Impact of genetic background and ablation of IRS-3 on IRS-2 knockout mice. Journal of Biological Chemistry 278 14284-14290.

Valet P, Tavernier G, Castan-Laurell I, Saulnier-Blache JS \& Langin D 2002 Understanding adipose tissue development from transgenic animal models. Journal of Lipid Research 43 835-860.

Zraika S, Dunlop M, Proietto J \& Andrikopoulos S 2002 Effects of free fatty acids on insulin secretion in obesity. Obesity Reviews 3 $103-112$.

Zraika S, Dunlop ME, Proietto J \& Andrikopoulos S 2004 Elevated SNAP-25 is associated with fatty acid-induced impairment of mouse islet function. Biochemical and Biophysical Research Communications 317 472-477.

Received 21 July 2005

Accepted 28 July 2005 